\title{
HIGH MORTALITY CAUSED BY BIBERSTEINIA TREHALOSI SEPTICAEMIA IN ADULT SHEEP - A CASE REPORT
}

\author{
Levente SzEREDI $^{1 *}$, Ferenc RAUSCH ${ }^{2}$, Zsófia SzELECZKY $^{1}$ and Szilárd JÁNOSI ${ }^{1}$ \\ ${ }^{1}$ Veterinary Diagnostic Directorate, Central Agriculture Office, Budapest, Hungary; \\ ${ }^{2}$ Veterinary Practitioner, Inárcs, Hungary
}

(Received 10 May 2018; accepted 25 July 2018)

\begin{abstract}
The disease induced by Bibersteinia trehalosi usually occurs in lambs. It is triggered by certain stress factors and often emerges in the form of severe outbreaks. In adult sheep, only sporadic cases have been reported so far. This paper reports a $B$. trehalosi-induced high-mortality case occurring only in adult sheep. Seventy out of 628 adult sheep (11\%) died in the affected pen during the six days of the outbreak. None of the 146 lambs kept in the neighbouring pen showed any clinical signs during that period. Several preceding events (shearing, vaccination and antiparasitic treatment) can be regarded as factors predisposing to the disease. Five adult sheep (4 females and 1 male) were sent for laboratory examination. Clinical, gross pathological, histological and bacteriological examinations revealed results corresponding to those reported previously in lambs that had died of a $B$. trehalosi-induced septicaemia.
\end{abstract}

Key words: Bibersteinia trehalosi, septicaemia, adult sheep, pathology, histology, immunohistochemistry

Pasteurellosis in sheep occurs in two clinical forms: pneumonia and systemic infection. Pneumonia is caused by Mannheimia haemolytica and Bibersteinia trehalosi in sheep of all ages. Septicaemia is induced by Bibersteinia trehalosi in 6- to 12-month-old lambs and by M. haemolytica in lambs less than 3 months of age. Both bacterial species are facultative pathogens and are normal inhabitants of the tonsils and nasopharynx of sheep (Donachie, 2000; Quinn et al., 2011). The first description of B. trehalosi-induced disease of lambs was published in 1955, in Scotland (Stamp et al., 1955), and the disease was subsequently diagnosed in other countries as well. In Hungary, a detailed description of the disease was first published in 1964 (Ratalics and Szabó, 1964). The occurrence of systemic $B$. trehalosi infection in lambs has been rarely reported in Hungary since that time (Hajtós et al., 1983, 1985; Szeredi et al., 2008).

In this paper we report a severe case of $B$. trehalosi-induced septicaemia occurring in a flock of adult sheep, leaving the lambs unaffected. To the best of our knowledge, this is the first report on the clinical, bacteriological, pathological, histological, and immunohistochemical diagnosis of this disease in adult sheep.

*Corresponding author; E-mail: szeredil@nebih.gov.hu 


\section{Materials and methods}

\section{Clinical history}

The affected flock was housed in a farm which had been used for this purpose in the preceding 16 years. Female $(n=616)$ and male $(n=12)$ breeding animals were kept in the same pen for the mating period, but were already separated prior to the outbreak. Animals intended for slaughter (200 animals) and yearlings (146 animals) were housed in two separate pens. Animals were treated with antiparasitics (Dectomax inj., Zoetis, and Vermitan oral, CEVA) on the 5th day after shearing in April 2017. In addition, the adult animals received vaccination as well (Coglamune vaccine, CEVA). The first clinical signs appeared in the breeding animals three days after the antiparasitic treatment and vaccination. A total of 70 breeding animals died during the six days of the outbreak. The clinical signs included fever, catarrhal nasal discharge, swallowing difficulty, diarrhoea, and abortions. In addition, several animals died suddenly, without showing any clinical signs. No disease was observed either in yearlings or in animals intended for slaughter. No similar disease was reported from the neighbouring flocks in that period of time.

\section{Gross pathological, histopathological and immunohistochemical examinations}

Five breeding sheep (4 female and 1 male) were sent for laboratory examinations. After dissection of the animals according to standard procedures at the laboratory, tissue samples from the liver, lungs and the brain were collected and fixed in $8 \%$ neutral buffered formalin (Reanal Co. Ltd., Budapest). The tissue samples were embedded in paraffin, sectioned at $4 \mu \mathrm{m}$, and stained using haematoxylin and eosin (HE) and Giemsa stain. Immunohistochemistry (IHC) was used on serial tissue sections to detect Bibersteinia antigens as reported previously (Szeredi et al., 2008; Ujvári et al., 2015).

\section{Bacteriological examinations}

Samples from the spleen, lungs and small intestinal content were streaked onto $10 \%$ sheep blood Columbia (Merck Kga, Germany) agar plates. Plates were incubated at $37^{\circ} \mathrm{C}$ for $24 \mathrm{~h}$ under aerobic conditions. In addition, plates from the small intestinal content were incubated at $37^{\circ} \mathrm{C}$ for $24 \mathrm{~h}$ under anaerobic conditions. Identification of the isolates was based on their colony morphology, Gramstaining profile and biochemical properties. Preliminary results were obtained with rapid tests, where we used commercially available tablets (Rosco Diagnostic tablets, BioConnections, UK) for the detection of carbohydrate fermentation and proteolytic enzymes, oxidase strips (Merck, Germany), indole spot reagent (Hardy Diagnostics, USA) and nitrate broth (in-house reagent). Further biochemical assays were performed with the GEN III MicroLog M System (Biolog, Inc., 
USA) according to the manufacturer's instructions. The plate was read visually and reactions were recorded using MicroLog M 5.2 software (Biolog, Inc. USA). The susceptibility of the isolated strain to antimicrobial drugs was measured with the agar disc diffusion method.

\section{Results}

\section{Gross pathological, histopathological and immunohistochemical examinations}

Gross pathological lesions were similar in all cases. Ecchymotic haemorrhages were evident in the subcutaneous tissues, and in the intermuscular fascia of the neck. Superficial lymph nodes were enlarged and congested. Petechial and ecchymotic haemorrhages were also observed in the pleura and the epicardium. Diffuse congestion was observed in the lungs, spleen, liver, and kidney. In two females well-developed twin lambs were found in the unaffected uterus. The livers of the pregnant animals were slightly yellowish. With the exception of one female with severe emaciation (Case 1), the animals showed good body condition. No other lesions could be detected.

The histological examination revealed a chronic multifocal, lungworminduced pneumonia in the female sheep presenting severe emaciation (Case 1). A few bacterial cells and a moderate amount of Bibersteinia antigens were found in the pulmonary vessels in this case. In the remaining four cases masses of bacteria and large amounts of Bibersteinia antigens were evident in the alveolar walls and lumen (Fig. 1A). A narrow, almost continual peripheral zone of acute tissue necrosis associated with these bacterial colonies could often be observed (Fig. 1B). Additionally, small bacterial clusters were observed in a few arterioles and veins. Small bacterial colonies were occasionally evident also in the lumen of bronchioles. Scant infiltrating inflammatory cells (lympho-histiocytic cells and neutrophil granulocytes) were rarely present around the bacterial colony. Additional lesions in the lungs were a diffuse, mild, lympho-histiocytic interstitial pneumonia associated with oedema, multifocal acute haemorrhages, and atelectasia, and the presence of acute thrombi in several blood vessels (Fig. 1B). In the liver, masses of bacteria and large amounts of Bibersteinia antigens were evident (Fig. 1C, D). Bacteria were present in the sinusoids and occasionally in hepatocytes (Fig. 1D). Bacterial colonies had a central strongly immunostained area, which was surrounded by a weakly labelled tissue area consisting of single bacterial cells (Fig. 1C). Bacterial colonies were frequently surrounded by a narrow continual peripheral zone of acute tissue necrosis (Fig. 1E, F). In addition, a mild lymphohistiocytic infiltration associated with oedema in the portal triad and the presence of acute thrombi occasionally incorporating bacterial cells (Fig. 1E, F) were observed in several blood vessels. In one female animal (Case 4) low to moderate numbers of infiltrating inflammatory cells were present around some bacterial colonies. With the exception of acute congestion, neither lesions nor bacterial 
colonies were evident in the brain. The HE and Giemsa staining and the IHC method had almost similar sensitivity in detecting bacterial colonies and cells (Table 1). However, the recognition of bacteria was much easier by the IHC technique compared to HE or Giemsa staining.

\section{Bacteriological examinations}

Large numbers of mucoid bacterial colonies grew out in pure culture on Columbia blood agar plates from the lungs and spleen samples in all cases. No bacterial growth was evident on Drigalski agar plates. The bacterium strains were identified as $B$. trehalosi according to their colony morphology, Gram staining and biochemical profile. The oxidase and nitrate reactions were positive, while urease, indole, catalase, ODC and ONPG tests were negative. According to the results of the Biolog GEN III MicroPlate system, the bacteria metabolised the following carbon sources: dextrin, D-maltose, D-trehalose, D-cellobiose, gentiobiose, sucrose, $\beta$-methyl-D-glucoside, D-salicin, N-acetyl-D-glucosamine, Nacetyl- $\beta$-D-mannosamine, $\mathrm{N}$-acetyl neuraminic acid, $\alpha$-D-glucose, D-mannose, D-fructose, inosine, D-sorbitol, D-mannitol, myo-inositol, glycerol, D-glucose-6PO4, D-fructose-6-PO4, D-aspartic-acid, L-alanine, L-aspartic acid, L-glutamic acid, L-pyroglutamic acid, L-serine, pectin, D-gluconic acid, methyl-pyruvate, Llactic acid, $\alpha$-ketoglutaric acid, L-malic acid, bromosuccinic acid, $\beta$-hydroxyD,L-butyric acid, $\alpha$-ketobutyric acid. The following carbon sources were not metabolised by the bacterial strain: D-raffinose, $\alpha$-D-lactose, D-melibiose, N-acetylD-galactosamine, D-galactose, 3-methyl-glucose, D-fucose, L-fucose, L-rhamnose, D-arabitol, D-serine gelatin, glycyl-L-proline, L-arginin, L-histidine, D-galacturonic acid, L-galactonic acid lactone, D-glucuronic acid, glucuronamide, mucic acid, quinic acid, D-saccharic acid, p-hydroxy-phenylacetic acid, D-lactic acid methyl ester, citric acid, D-malic acid, Tween 40, $\gamma$-amino-butyric acid, $\alpha$-hydroxybutyric acid, propionic acid, formic acid, while the results of D-turanose, stachyose and acetic acid tests were borderline. There was growth in 1\% sodium lactate, Rifamycin SV, vancomycin, nalidixic acid, tetrazolium blue and potassium tellurite, and no growth in $\mathrm{pH} 6, \mathrm{pH} 5 \mathrm{NaCl}(1 \%, 4 \%$ and $8 \%)$, fusidic acid, troleandomycin, minocycline, lincomycin, guanidine $\mathrm{HCl}$, Niaproof 4 , tetrazolium violet, lithium chloride, aztreonam, sodium butyrate, and sodium bromate. The strain was susceptible to penicillin, amoxicillin, ampicillin, amoxicillin and clavulanic acid, gentamicin, streptomycin, enrofloxacin, marbofloxacin, flumequine, tetracycline, doxycycline, erythromycin, tulathromycin, sulphonamides, florfenicol, cephalexin, ceftiofur, cefquinom and moderately sensitive to spectinomycin. Additionally, moderate numbers of bacterial colonies of Clostridium perfringens were isolated from the small intestinal content in four cases (Cases 1, 3, 4 and 5). 

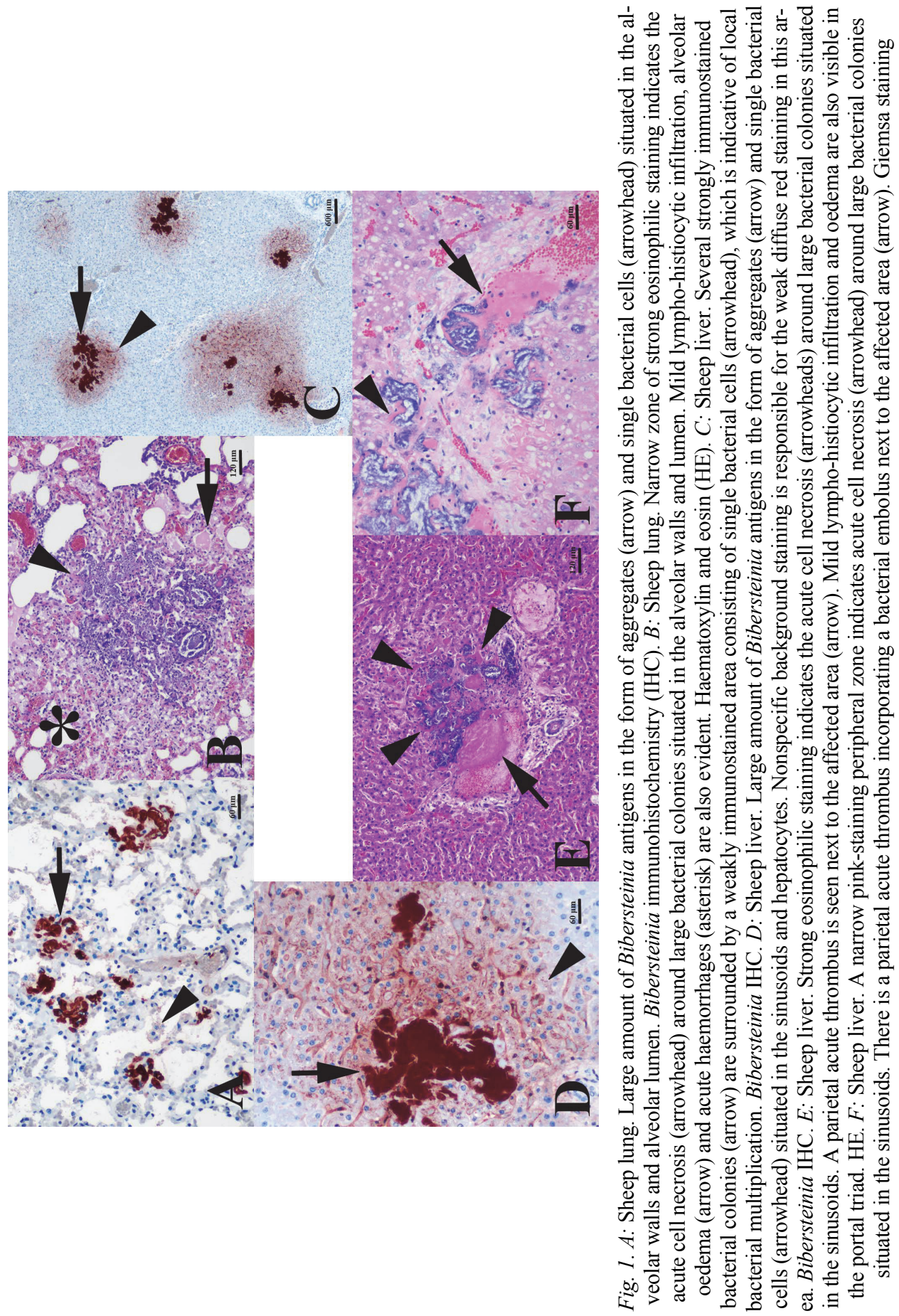


\section{Table 1}

The presence of characteristic histological lesions and the number of bacterial colonies using haematoxylin and eosin (HE) and Giemsa staining, and the amount of Bibersteinia antigens detected by immunohistochemistry (IHC) in tissue samples of breeding animals

\begin{tabular}{|c|c|c|c|c|c|}
\hline Case number ${ }^{*}$ & Organ & $\begin{array}{l}\text { Histological } \\
\text { lesions }^{* *}\end{array}$ & $\begin{array}{c}\text { HE } \\
\text { staining }^{* * *}\end{array}$ & $\begin{array}{l}\text { Giemsa } \\
\text { staining }^{* * *}\end{array}$ & $\mathrm{IHC}^{* * *}$ \\
\hline \multirow[t]{3}{*}{ Case 1} & lungs & - & + & + & ++ \\
\hline & liver & + & ++ & ++ & +++ \\
\hline & brain & - & - & - & - \\
\hline \multirow[t]{3}{*}{ Case 2} & lungs & + & +++ & +++ & +++ \\
\hline & liver & + & +++ & +++ & +++ \\
\hline & brain & - & - & - & - \\
\hline \multirow[t]{3}{*}{ Case 3} & lungs & + & +++ & +++ & +++ \\
\hline & liver & + & +++ & +++ & +++ \\
\hline & brain & - & - & - & - \\
\hline \multirow[t]{3}{*}{ Case 4} & lungs & + & +++ & +++ & +++ \\
\hline & liver & + & +++ & +++ & +++ \\
\hline & brain & - & - & - & - \\
\hline \multirow[t]{3}{*}{ Case 5} & lungs & + & +++ & +++ & +++ \\
\hline & liver & + & +++ & +++ & +++ \\
\hline & brain & - & - & - & - \\
\hline
\end{tabular}

${ }^{*}$ Cases 1-4: females, Case 5: male; ${ }^{* *}$-: no characteristic lesions, +: characteristic lesions present; *** -: no bacteria or Bibersteinia antigens, +: low number of bacterial colonies, ++ : moderate amount of Bibersteinia antigens, +++: large number of bacterial colonies and large amount of Bibersteinia antigens

\section{Discussion}

Septicaemia induced by $B$. trehalosi was shown to be associated with different stress factors such as weaning, adverse climatic conditions, transportation, vaccination, antiparasitic treatment, and shearing (Stamp et al., 1955; Dyson et al., 1981; Hajtós et al., 1985; Mackie et al., 1995). Even a change in feeding (moving from poor-quality pasture to a succulent pasture in the autumn, or a poor-quality feed) was found to be associated with the disease (Stamp et al., 1955; Dyson et al., 1981; Hajtós et al., 1985; Mackie et al., 1995). In the present study the disease was also associated with different predisposing factors (shearing, antiparasitic treatment, and vaccination). Interestingly, only adult animals and no lambs were affected in this outbreak, which is in contrast to earlier reports. We could not find any explanation for this phenomenon. There has been a single study reporting the occurrence of the disease in adult sheep (Hajtós et al., 1985). In that case 9 out of 400 animals (2\%) died three days after receiving an antiparasitic treatment. The $B$. trehalosi-induced outbreak was more severe in the 
present study, causing the death of $70(11 \%)$ animals within a few days in the group of breeding animals. A possible explanation for that might be that the $B$. trehalosi strain associated with this outbreak differed in pathogenicity from those involved in the previously published cases. It is important to note that the antiparasitic treatment and the vaccination were performed by the owner, and thus iatrogenic infection could be not completely excluded.

The gross pathological and histological alterations were similar to those reported previously in lambs. The main gross pathological lesion was the presence of haemorrhages in the subcutaneous tissue and intermuscular fascia of the neck, in the pleura and the epicardium. The histological lesions were characterised by a widespread bacterial embolism often associated with a narrow peripheral zone of acute necrosis, while inflammatory cell infiltrates around the bacteria were absent or minimal. Earlier reports indicated that $B$. trehalosi induces a systemic disease in lambs with bacterial multiplication occurring primarily in organs, rather than in the blood, the latter being characteristic of septicaemia (Dyson et al., 1981). However, recent results in human medicine have demonstrated that septicaemia, often called 'blood poisoning', can be regarded as the reaction of the host organism to different kinds of infections, and it is not inevitably associated with the multiplication of the pathogenic organisms in the blood (reviewed by Lucas, 2012; Angus and van der Poll, 2013). Accordingly, the B. trehalosiinduced disease of sheep should be accepted as septicaemia despite the fact that bacterial multiplication occurs primarily in the organs. The sudden death, the short clinical course and the presence of different histological alterations (acute thrombi, acute tissue necrosis and acute haemorrhages) supported the diagnosis of septicaemia in the present case. The results of IHC test further supported the occurrence of local bacterial multiplication based on the detection of a wide peripheral zone containing single bacterial cells around a central strongly immunostained area; the latter might have originated from a previous bacterial dissemination (Fig. 1C).

The detection of Pasteurella antigens using IHC method was suggested to be of equal value as, or superior to, bacterial culture in pneumonia of cattle or septicaemia of swine (Haritani et al., 1987; Ono et al., 2003). This technique was helpful also in the present study, indicating the distribution of the causative bacterium in several organs. In this case, the histochemical staining methods (HE, Giemsa) were almost similarly sensitive for the detection of bacteria in tissue sections, because of the presence of large numbers of bacteria in the affected tissues. However, significantly more bacterial cells could be detected by the IHC method than by HE and Giemsa staining.

The clinical signs in the present study were similar to those reported previously (Stamp et al., 1955; Ratalics and Szabó, 1964; Hajtós et al., 1983). However, clinical signs alone are not pathognomonic for the disease, hence laboratory examinations are essential for the definitive diagnosis. The results of gross 
pathological and histological examinations and identification of the causative bacterium species using classical bacteriological methods provided a safe diagnosis in the present study. No other causative infections, including bluetongue disease, were detected (data not shown). Animals showing clinical signs were treated with different antibiotics (tulathromycin, cefquinome, and enrofloxacin) in the present case, unfortunately with only limited success (data not shown). Similar results were published previously (Ratalics and Szabó, 1964).

In conclusion, the disease induced by $B$. trehalosi can cause a high mortality rate not only in lambs but in adult sheep as well. The presence of different predisposing factors in a flock is primarily responsible for the disease of adult animals, in the same way as in the case of lambs. Antibiotics have limited efficacy in the treatment of affected breeding animals.

\section{Acknowledgement}

The technical assistance of Mrs É. Gégényiné is gratefully acknowledged.

\section{References}

Angus, D. C. and van der Poll, T. (2013): Severe sepsis and septic shock. N. Engl. Med. 369, $840-851$.

Donachie, W. (2000): Pasteurellosis. In: Martin, W. B. and Aitken, I. D. (eds) Diseases of Sheep. 3rd edition. Blackwell Science Ltd., Oxford, UK. pp. 191-198.

Dyson, D. A., Gilmour, N. J. L. and Angus, K. W. (1981): Ovine systemic pasteurellosis caused by Pasteurella haemolytica biotype T. J. Med. Microbiol. 14, 89-95.

Hajtós, I., Fodor, L., Varga, J. and Malik, G. (1985): Incidence of Pasteurella haemolytica serotypes and associated diseases in sheep [in Hungarian, with English abstract]. Magy. Allatorvosok 40, 473-479.

Hajtós, I., Harrach, B., Szigeti, G., Fodor, L., Malik, G. and Varga, J. (1983): Stachybotryotoxicosis as a predisposing factor of ovine systemic pasteurellosis. Acta Vet. Hung. 31, 181-188.

Haritani, M., Nakazawa, M., Oohashi, S., Yamada, Y., Haziroglu, R. and Narita, M. (1987): Immunoperoxidase evaluation of pneumonic lesions induced by Pasteurella haemolytica in calves. Am. J. Vet. Res. 48, 1358-1362.

Lucas, S. (2012): The autopsy pathology of sepsis-related death. In: Fernandez, R. (ed.) Severe Sepsis and Septic Shock - Understanding a Serious Killer. InTech, Rijeka, Croatia. pp. 71-100. (http://www.intechopen.com/books/severe-sepsis-and-septic-shock-understanding-aserious-killer/theautopsy-pathology-of-sepsis-and-septic-shock)

Mackie, J. T., Barton, M. and Hindmarsh, M. (1995): Pasteurella haemolytica septicaemia in sheep. Aust. Vet. J. 72, 474.

Ono, M., Okada, M., Namimatsu, T., Fujii, S., Mukai, T. and Sakano, T. (2003): Septicaemia and arthritis in pigs experimentally infected with Pasteurella haemolytica capsular serotype A. J. Comp. Pathol. 129, 251-258.

Quinn, P. J., Markey, B. K., Leonard, F. C., FitzPatrick, E. S., Fanning, S. and Hartigan, P. J. (2011): Pasteurella species, Mannheimia haemolytica and Bibersteinia trehalosi. In: Veterinary Microbiology and Microbial Disease. 2nd edition. Blackwell Science Ltd., Oxford, UK. pp. 300-308. 
Ratalics, L. and Szabó, J. (1964): Disease of sheep caused by Pasteurella haemolytica [in Hungarian]. Magy. Allatorvosok 19, 112-114.

Szeredi, L., Csabai, T., Makrai, L. and Jánosi, Sz. (2008): Immunohistochemical detection of Bibersteinia (Pasteurella) trehalosi in a lamb died due to acute systemic infection [in Hungarian, with English abstract]. Magy. Allatorvosok 130, 658-662.

Stamp, J. T., Watt, J. A. A. and Thomlinson, J. R. (1955): Pasteurella haemolytica septicaemia of lambs. J. Comp. Pathol. 65, 183-196.

Ujvári, B., Szeredi, L., Pertl, L., Tóth, G., Erdélyi, K., Jánosi, Sz., Molnár, T. and Magyar, T. (2015): First detection of Pasteurella multocida type B:2 in Hungary associated with systemic pasteurellosis in backyard pigs. Acta Vet. Hung. 63, 141-156. 